\title{
PERFIL DOS DEPENDENTES QUÍMICOS ATENDIDOS EM UMA UNIDADE DE REABILITAÇÃO DE UM HOSPITAL PSIQUIÁTRICO
}

The profile of the chemical dependants assisted in a rehabilitation unit of a psychiatric hospital

Perfil de los dependientes químicos atendidos en una unidad de rehabilitación de un hospital psiquiátrico

Luiz Henrique Prado da Silva ${ }^{1}$

Andréa Noeremberg Guimarães ${ }^{4}$

\author{
Letícia de Oliveira Borba ${ }^{2}$
}

Maria de Fátima Mantovani ${ }^{5}$
Marcio Roberto Paes ${ }^{3}$

Mariluci Alves Maftum ${ }^{6}$

\section{RESUMO}

Pesquisa descritiva. Objetivo: caracterizar o perfil dos dependentes químicos atendidos na unidade de reabilitação de um hospital psiquiátrico. Participaram 30 dependentes químicos. Os dados foram coletados mediante entrevista. A faixa etária prevalente foi entre 26 e 33 anos: $50 \%$ estavam empregados; $77 \%$ perderam o emprego pelo menos uma vez pelo abuso de drogas; $80 \%$ tiveram episódios de separação relacionada com o uso de drogas; 11 participantes tinham diagnóstico de transtorno mental antes do internamento e, destes, 9 tentaram suicídio; $71 \%$ iniciaram o uso de drogas pelo álcool com maior prevalência na faixa etária dos 12 a 19 anos; $71 \%$ tiveram contato com a droga no meio familiar e $30 \%$ dos casos, por meio de amigos. 0 abuso dessas substâncias afeta pessoas em idade produtiva, adultos jovens, e prejudica o desempenho no trabalho e na relação familiar.

Palavras-chave: Saúde Mental. Enfermagem. Drogas.

\begin{abstract}
Descriptive research with the aim of characterizing the profile of chemical dependants assisted in the rehabilitation unit of a psychiatric hospital. Thirty chemical dependants joined the research. Data were collected from an interview. The prevalent age group was from 26 to 33 years old, $50 \%$ of them were unemployed; $77 \%$ lost their jobs at least once caused by the drug abuse; $80 \%$ had an episode of separation related with drug use; 11 participants had a metal problem diagnosed before the hospitalization, and among these, 9 tried to commit suicide.; $71 \%$ began the drug use by taking alcohol and by the age of 12 to 19 years old; $71 \%$ had their first contact with drugs among family members and in $30 \%$ of the cases it was through friends. The abuse of these substances affects people in productive age, young adults which begun the use among family sets, and affected their work performance and family relationship.
\end{abstract}

Keywords: Mental Health. Nursing. Drugs.

\section{Resumen}

Investigación descriptiva. Objetivo: caracterizar el perfil de los dependientes químicos atendidos en la unidad de rehabilitación de un hospital psiquiátrico. Participaron 30 dependientes químicos. Los datos fueron colectados por medio de entrevista. El grupo etario prevalente fue entre 26 y 33 años, $50 \%$ empleados; $77 \%$ perdió el empleo por lo menos una vez por abuso de drogas; el $80 \%$ tuvo episodios de separación relacionada con el uso de drogas; 11 participantes fueron diagnosticados con trastorno mental antes de la internación y de estos, 9 intentaron el suicidio; $71 \%$ inició el uso de drogas por el alcohol con mayor predominancia en el rango de edad de 12 hasta 19 años; $71 \%$ tuvo contacto con la droga en el ambiente familiar y en $30 \%$ de los casos fue a través de amigos. El abuso de esas sustancias afecta personas en edad productiva, adultos jóvenes, cuyo inicio ocurrió en el círculo familiar y afectó el desempeño en el trabajo y la relación familiar del usuario.

Palabras clave: Salud Mental. Enfermería. Drogas.

\footnotetext{
${ }^{1}$ Acadêmico do Curso de Graduação em Enfermagem da Universidade Federal do Paraná - UFPR.Curitiba-PR- Brasil. E-mail: enjoyluizufpr@hotmail.com., ${ }^{2}$ Enfermeira. Mestranda do Programa de Pós-Graduação em Enfermagem da UFPR. Membro do Núcleo de Estudos, Pesquisa e Extensão em Cuidado Humano de Enfermagem (NEPECHE/UFPR). Bolsista CAPES.Curitiba-PR-Brasil. E-mail: leticia_ufpr@yahoo.com.br., ${ }^{3}$ Enfermeiro. Mestrando do Programa de Pós-Graduação em Enfermagem da UFPR. Membro do NEPECHE/UFPR.Curitiba-PR-Brasil. E-mail: marropa@pop.com.br., ${ }^{4}$ Enfermeira. Mestranda do Programa de Pós-Graduação em Enfermagem da UFPR. Membro do NEPECHE/UFPR.Curitiba-PR- Brasil. E-mail: deia@ufpr.br, ,5outora em Enfermagem. Docente e Vice-coordenadora do Curso de Mestrado em Enfermagem do Programa de Pós-Graduação em Enfermagem da UFPR. Coordenadora do Grupo de Estudos Multiprofissional em Saúde do Adulto (GEMSA/ UFPR).Curitiba-PR- Brasil. E-mail: mantovan@ufpr.br., ${ }^{6}$ Doutora em Enfermagem. Docente do Curso de Mestrado em Enfermagem do Programa de Pós-Graduação em Enfermagem da UFPR. Vice-coordenadora do NEPECHE/UFPR.Curitiba-PR- Brasil. E-mail: maftum@ufpr.br
} 


\section{INTRODUÇÃO}

0 uso de substâncias psicoativas é uma prática antiga e presente em várias culturas desde os tempos pré-históricos; portanto, faz parte da história da humanidade. Alguns povos faziam uso dessas substâncias para fins terapêuticos, outros as usavam em seus ritos religiosos. As substâncias psicoativas eram utilizadas por cada sociedade conforme sua cultura, época, seus conhecimentos, e, ainda hoje, seu consumo se encontra bastante evidente e comum em algumas sociedades. ${ }^{1-2}$

Historicamente, as culturas primitivas atribuíam o uso de sustâncias psicoativas a uma necessidade religiosa ou medicinal e, dessa maneira, era permitido o consumo criterioso da substância, o que reduzia em grande parte seu potencial de abuso. Entretanto, o uso de substâncias psicoativas está cada vez mais disseminado nas sociedades, é comum se deparar com a notícia de que um amigo ou familiar se tornou dependente químico. Ao se considerar que grande parte das sociedades tenha recorrido ao uso de algum tipo de substância psicoativa, é possível que esse consumo esteja ligado ao convívio familiar e social. Mesmo que por diferentes motivos, se pode afirmar que a sua utilização se trata de uma prática humana, milenar e universal. ${ }^{1,3-4}$

Um marco importante na história contemporânea das substâncias psicoativas é a caracterização da dependência química como transtorno mental, o que possibilita, além de tratar os dependentes químicos, investir na desestigmatização que eles sofrem. ${ }^{2}$ Esse deve ser entendido como uma pessoa com uma doença multicausal que necessita no curso de seu tratamento, de um trabalho multiprofissional com intervenç̃̃es que visem sua reinserção à sociedade e o retorno ao seu estado sadio. Assim, deve-se, preferencialmente, tratá-lo em seu meio social, porém tão importante quanto tratá-lo é investir em estratégias de promoção da saúde e prevenção do uso e abuso de drogas. ${ }^{4.5}$

A complexidade do tema relacionado ao uso de substâncias psicoativas é refletida na terminologia associada, que parece mudar toda vez que comitês profissionais e governamentais se reúnem para discutir o problema. Uma das dificuldades é como denominar as substâncias que alteram as funções cerebrais. No Brasil, para classificar os transtornos decorrentes do uso de substâncias psicoativas, adota-se os conceitos de maior aceitação internacional, os da Classificação Estatística Internacional de Doenças e Problemas relacionados com a Saúde da CID-10.2

A dependência química é um transtorno heterogêneo, visto que atinge pessoas de diferentes maneiras, afeta tanto seu corpo físico como suas relações interpessoais, por diversas razões, em diferentes meios e circunstâncias. A sociedade, ao isolar culturalmente o usuário de drogas, faz com que muitos destes não compartilhem da expectativa e desejo de abstinência com os profissionais de saúde e sequer procurem atendimento, pois não se sentem acolhidos em suas diferenças. ${ }^{6}$
A questão do uso abusivo de álcool e outras drogas tem sido tratada, predominantemente, sob o ponto de vista biomédico, centrado na doença e na cura. Entretanto, as implicações sociais, psicológicas, econômicas e políticas são evidentes e devem ser consideradas na compreensão global do problema. $^{6}$

0 uso indiscriminado de substâncias psicoativas vem sendo associado à criminalidade e às práticas antissociais relacionadas ao comportamento irresponsável do usuário, que acaba por cometer atos de delinquência e envolver-se com problemas de ordem judiciária. Isso acarreta perdas individuais e sociais, o que leva o dependente à exclusão social. ${ }^{1}$

Os profissionais da área da saúde devem dar importância ao componente físico no momento da avaliação do usuário de substância psicoativa. Também, devem desenvolver competências básicas para atender essa demanda, pois, quando a dependência química não é detectada, ela pode acarretar problemas clínicos, psiquiátricos e sociais. ${ }^{7}$ Ressalta-se que 0 planejamento das ações nos serviços de reabilitação devem ser voltados à atenção integral dessa população, visto que a política nacional do Brasil preconiza e assegura aos dependentes químicos o direito a um modelo de tratamento integrado e descentralizado. ${ }^{6}$

Pode-se considerar a dependência química um grave problema de saúde pública, haja vista as implicações que o uso, o abuso e a dependência de drogas acarretam para a pessoa e para a sociedade. 0 último relatório apresentado pela Organização das Nações Unidas (ONU) com dados de 2007 mostra que, aproximadamente, 208 milhões de pessoas (4,8\% da população adulta do mundo) usaram drogas ilíitas ao menos uma vez naquele ano. Também demonstra que 26 milhões ( $0,6 \%$ da população mundial) têm dependência química e que metade faz uso de drogas pelo menos uma vez ao mês, e, em média, cerca de 200 mil usuários morreram no ano de 2007 em consequência do consumo, e outros tantos desenvolveram comorbidades. ${ }^{8}$

A procura por tratamento entre adultos, jovens e adolescentes tem aumentado na última década. Observase o surgimento de diversificados tipos de drogas, o que muda o modo e o tipo de consumo. ${ }^{9}$ Diante dessa problemática atual em que cada vez mais pessoas, principalmente os jovens, se envolvem com a drogadição fica evidente a importância de estudos nessa temática, bem como conhecer o perfil dos dependentes químicos que buscam auxílio em unidades de reabilitação. Destarte, 0 conhecimento sobre o perfil dos clientes com dependência química pode auxiliar na elaboração de estratégias de prevenção e tratamento, buscando integrar profissionais de saúde, família, usuário e sociedade.

Portanto, nesse estudo, tem-se o objetivo de caracterizar o perfil dos dependentes químicos atendidos na unidade de reabilitação de um hospital psiquiátrico. 


\section{MATERIAIS E MÉTODO}

Trata-se de uma pesquisa descritiva, com abordagem quantitativa, desenvolvida no período de setembro a novembro de 2008, em uma Unidade de Reabilitação de Adictos (URA) de um hospital especializado em psiquiatria de um município do Paraná. Esta unidade interna pacientes do sexo masculino para tratamento de alcoolistas e drogaditos e conta com 30 leitos. 0 tempo máximo de internamento na URA é de 30 dias, sendo que após o trigésimo dia a equipe multiprofissional faz a avaliação do dependente e o prazo pode se estender por mais 15 dias, totalizando 45 dias.

A amostra do estudo foi composta por 30 dependentes químicos, maiores de 18 anos, internados na URA, que aceitaram participar e assinar o Termo de Consentimento Livre e Esclarecido.

Os princípios éticos atendendo a Resolução n.196/96 do Conselho Nacional de Saúde foram respeitados mediante informações e esclarecimentos de todas as etapas do estudo aos participantes e quanto à liberdade de participar ou desistir a qualquer momento. Também foi garantido o direito à privacidade, 0 livre acesso aos dados, o anonimato. 0 projeto foi aprovado pelo Comitê de Ética do Setor de Ciências da Saúde da Universidade Federal do Paraná/UFPR, em 09/ 09/2008 sob o Registro CEP/SD: 609.146.08.08. CAAE: 2726.0.000.091-08. ${ }^{10}$

Os dados foram coletados mediante a técnica da entrevista estruturada cujo instrumento foi composto de duas partes: a primeira se destinou à coleta dos dados de identificação e a segunda, de informações a respeito do uso de drogas pelo participante. Os dados foram tabulados em planilhas do programa Microsoft Office Excel ${ }^{\circledR}$ e são apresentados em tabelas com números absolutos e percentuais.

\section{RESULTADOS E DISCUSSÃO}

$\mathrm{Na}$ Tabela 1, são apresentados dados de caracterização dos dependentes químicos que participaram desta pesquisa, referentes à procedência e à idade.

Tabela 1 - Distribuição dos usuários de drogas da URA segundo procedência e idade. Paraná, 2008

\begin{tabular}{cccccccc}
\hline Procedência & $\begin{array}{c}\text { Curitiba \% } \\
\text { Faixa Etária }\end{array}$ & $\begin{array}{c}\text { Interior do } \\
\text { Estado\%(n) }\end{array}$ & $\begin{array}{c}\text { Região } \\
\text { Metropolitana\%(n) }\end{array}$ & $\begin{array}{c}\text { Outros } \\
\text { Estados } \\
\text { \%(n) }\end{array}$ & $\begin{array}{c}\text { Total } \\
\text { \% (n) }\end{array}$ \\
\hline $\mathbf{1 8 - 2 5}$ & & $3(1)$ & $17(4)$ & & 16 & $(5)$ \\
$\mathbf{2 6 - 3 3}$ & $10(3)$ & $10(3)$ & $17(5)$ & & 37 & $(11)$ \\
$\mathbf{3 4 - 4 0}$ & $7(2)$ & $3(1)$ & $7(2)$ & 3 & $(1)$ & 20 & $(6)$ \\
$\mathbf{4 1 - 4 8}$ & $3(1)$ & & $7(2)$ & & 10 & $(3)$ \\
$\mathbf{4 9 - 5 6}$ & & & $7(2)$ & & 7 & $(2)$ \\
$\mathbf{5 7 - 6 4}$ & $3(1)$ & & $7(2)$ & & 10 & $(3)$ \\
\hline Total & $23(7)$ & $16(5)$ & $58(17)$ & 3 & $(1)$ & 100 & $(30)$ \\
\hline
\end{tabular}

Fonte: Da pesquisa

Verificou-se que a maioria $81 \%(n=24)$ é procedente de Curitiba e Região Metropolitana. Os dados encontrados nesta pesquisa demonstram que 0 abuso de substâncias psicoativas atinge indivíduos de diferentes idades, e que há um acréscimo nos usuários com idade entre 26 e 33 anos - 37\% $(n=11)$ e que $16 \%(n=5)$ encontram-se na faixa etária de 18 a 25 anos. A expectativa para essa faixa etária é que os indivíduos estivessem em formação escolar ou iniciando sua vida no mundo do trabalho; porém com o uso abusivo das substâncias psicoativas, eles comumente deixam de estudar e compõem o grupo de risco para o desemprego, geralmente estão respondendo por problemas legais, se envolvem com acidentes automobilísticos, atos violentos e suicídio. ${ }^{1,9}$
Esses usuários foram encaminhados a URA para internação por meio de um Centro de Atenção Psicossocial (CAPSad). Isso demonstra um avanço nos modos de tratamento, sendo contemplado serviço de atenção básica aos usuários de álcool e outras drogas de acordo com as normas estabelecidas pelo Ministério da Saúde e o preconizado pela Reforma Psiquiátrica Brasileira. ${ }^{6}$

Os achados corroboram os dados da ONU de que cerca de $10 \%$ das populações que vivem nos grandes centros urbanos utilizam de forma abusiva algum tipo de substância psicoativa, indiferente do sexo, idade, nível de escolaridade, poder aquisitivo e condição de moradia. ${ }^{8}$ 
Tabela 2 - Grau de escolaridade e situação de emprego dos dependentes químico da URA. Paraná, 2008

\begin{tabular}{|c|c|c|c|c|c|}
\hline Escolaridade & \multirow{2}{*}{$\begin{array}{l}\text { Analfabeto } \\
\% \quad \text { (n) }\end{array}$} & \multirow{2}{*}{$\begin{array}{c}\text { Ensino } \\
\text { Fundamental } \\
\text { Completo } \\
\begin{array}{cc}\% & \text { (n) }\end{array}\end{array}$} & \multirow{2}{*}{$\begin{array}{l}\text { Ensino } \\
\text { comple to } \\
\text { Médio } \\
\% \quad \text { (n) }\end{array}$} & $\begin{array}{c}\text { Ensino } \\
\text { Superior }\end{array}$ & Total \\
\hline $\begin{array}{l}\text { Situação de } \\
\text { emprego }\end{array}$ & & & & $\begin{array}{l}\text { Superior } \\
\% \quad \text { (n) }\end{array}$ & \\
\hline Autônomo & (2) & $\begin{array}{ll}3 & (2)\end{array}$ & $20 \quad(6)$ & (2) & $37 \quad(11)$ \\
\hline Desemnregado & & 7 (1) & & & 7 (2) \\
\hline Estudante & & (1) & & & $3 \quad$ (1) \\
\hline Anosentado & & & (1) & & 3 (1) \\
\hline CLT & & $\begin{array}{ll}30 & (9) \\
\end{array}$ & $13 \quad(4)$ & (2) & $50 \quad(15)$ \\
\hline Total & (2) & $43 \quad(13)$ & $36 \quad(11)$ & $14 \quad(4)$ & $100 \quad(30)$ \\
\hline
\end{tabular}

Fonte: Da pesquisa.

Os dados da Tabela 2 evidenciaram que as drogas atingem pessoas com diferentes graus de escolaridade e situação empregatícia, sendo que somente $7 \%(n=2)$ dos respondentes são analfabetos, $30 \%(n=9)$ possuem o ensino fundamental completo, e o dobro $14 \%(n=4)$ possui curso superior completo. Ressalta-se que $50 \%(n=15)$ tinham emprego com carteira assinada e estavam trabalhando antes de serem encaminhados à internação na URA, e apenas $7 \%$ $(n=2)$ estavam desempregados.

Questionados sobre o impacto das drogas em sua vida profissional, a maioria, $77 \%$ ( $n=23$ ), afirmou que perdeu 0 emprego pelo menos uma vez devido ao abuso de substâncias psicoativas. Os usuários de substâncias sofrem perdas de ordem física e psíquica. ${ }^{11}$

Quanto ao estado civil, $56 \%(n=17)$ são solteiros; $23 \%(n=7)$, casados; $18 \%(n=5)$, amasiados; e 3\% $(n=1)$, viúvos. Tem-se que $80 \%$ ( $n=24$ ) da amostra já moraram com companheira e relataram que a separação teve relação direta com a drogadição.

Viver com um dependente químico não constitui tarefa fácil, pois são frequentes as brigas familiares e, consequentemente, os divórcios, uma vez que o usuário de droga pensa, na maioria das vezes, de modo egoísta quando está sob o efeito da droga e tem o pensamento apenas voltado ao consumo da substância pela qual está dependente. ${ }^{12}$ Ainda, o usuário de substância psicoativa tem perdas individuais como: perda do emprego, bens pessoais, prejuízos à saúde e rompimento do vínculo familiares. Brigas entre dependentes químicos e familiares são constantes, e isso leva ao divórcio e a perda do contato com os filhos, muitas vezes estabelecida por um juiz a pedido da mãe. ${ }^{13}$

Tabela 3 - A relação do transtorno mental e tentativas de suicídio dos dependentes químicos da URA. Paraná, 2008

\begin{tabular}{|c|c|c|c|c|}
\hline \multirow{2}{*}{$\begin{array}{c}\text { Tentativa de suicídio } \\
\text { Transtorno }\end{array}$} & \multirow{2}{*}{${ }_{\%}$ Sim $^{\text {Sn) }}$} & \multirow{2}{*}{$\begin{array}{l}\text { Não } \\
\% \text { (n) }\end{array}$} & \multicolumn{2}{|c|}{ Total } \\
\hline & & & $\%$ & (n) \\
\hline Transtorno Afetivo-Binolar & (3) & & 10 & (3) \\
\hline Depressão & $27 \quad(8)$ & (19) & 90 & $(27)$ \\
\hline Total & $37 \quad(11)$ & (19) & 100 & (30) \\
\hline
\end{tabular}

ronte: va pesquisa.

A Tabela 3 mostra que 37\% $(n=11)$ da amostra já haviam apresentado algum transtorno mental antes do internamento atual; destes, $10 \%(n=3)$ referiram transtorno afetivo bipolar e $27 \%(n=8)$, algum quadro de depressão com diagnóstico médico estabelecido. Outro dado relevante encontrado nesta pesquisa se refere a $30 \%(n=9)$ dos sujeitos que possuem algum transtorno mental associado à dependência química; já tentaram suicídio por no mínimo uma vez na vida e a maior prevalência é daqueles que tem depressão.

Ressalta-se que o transtorno mental por si só já é fator de risco para o suicídio, e, quando há a comorbidade com a dependência química, esse risco é potencializado. As substâncias psicoativas exacerbam ou predispõem os indivíduos a qualquer outro tipo de transtorno mental, principalmente aqueles relacionados com o humor e 0 afeto. ${ }^{2}$

0 suicídio deve ser reconhecido como prioridade em saúde pública, pois se apresenta entre as dez principais causas de morte em todas as idades e entre as três primeiras na faixa etária de 15 a 35 anos. 0 uso abusivo de álcool e outras drogas é fator predisponente para o suicídio, e a prevalência da tentativa de suicídio é maior em usuários de substâncias psicoativas. Um estudo ${ }^{14}$ quantificou as tentativas de suicídio em um hospital geral e observou que $15 \%$ estavam relacionadas ao uso de álcool, $11 \%$ ao uso de drogas ilíitas e $27 \%$ ao uso de medicamentos psicoativos. 
Outro estudo do Programa de Monitoramento de Uso de Drogas, realizado em Nova lorque, entre os anos de 1997 e 2007, indicou que $35 \%$ de todos os adolescentes detidos reportaram algum envolvimento com álcool, 70\% com drogas e $75 \%$ com álcool e drogas. ${ }^{13}$ Salvaguardadas as devidas proporções, constatou-se nesta pesquisa que na URA $88 \%$ $(n=26)$ dos dependentes já tiveram problemas judiciais, pelo envolvimento com a droga e destes, $77 \%(n=23)$ foram relacionados ao uso de cracke $11 \%(n=3)$, ao de álcool.

Os sujeitos da pesquisa, ao serem questionados a respeito do tipo de drogas que já consumiram no decorrer de suas vidas, referiram: $87 \%(n=26)$ álcool, $83 \%(n=21)$ maconha, $73 \%(n=22)$ crack, $70 \%(n=21)$ cocaína, $60 \%$ $(n=18)$ tabaco, 30\% ( $n=9)$ ecstasy, e 67\% ( $n=20)$ utilizaram outras drogas, como LSD, chá de cogumelo, rebite, heroína, tinger e medicamentos. Constata-se que o maior consumo de droga lícita é do álcool, seguido pelo tabaco, e, do grupo das drogas ilícitas, a maconha, o cracke a cocaína continuam sendo as drogas de uso mais prevalente.

Por fatores históricos e culturais, o álcool sempre foi a droga lícita com maior difusão entre os povos. Não é difícil observar jovens menores de 18 anos que usam essa droga em festas, bares ou até mesmo dentro de casa com prévia aprovação dos pais. ${ }^{2}$ As drogas de modo geral, principalmente o álcool, são intrínsecas à nossa cultura e por vários motivos são consumidas. ${ }^{1}$ É a droga que leva o maior número de indivíduos ao tratamento especializado, porém com o avanço do tráfico e a maior variabilidade de drogas ofertadas, esse quadro tende a mudar nos próximos anos. Mesmo assim, o álcool será a droga de maior consumo por ser lícita, mas as causas de internamentos poderão se equivaler às de outras drogas. ${ }^{15}$

0 álcool e o tabaco, por serem drogas lícitas, podem ser obtidos e utilizados de maneira mais fácil. Como essas drogas causam danos físicos e psicológicos de maneira muito lenta, é comum encontrar dependentes que as usam por 20, 30 anos. Por outro lado, a cocaína e o cracksão drogas ilícitas e com maior dificuldade de obtenção e uso; agridem de forma mais violenta o usuário e, portanto, é menos provável encontrar alguém que os utiliza por longo período de tempo. Normalmente aqueles que fazem uso abusivo dessas substâncias apresentam comorbidades graves ou morrem devido ao uso excessivo, ${ }^{2,13}$

Merece destaque o fato de o Brasil possuir o segundo maior mercado de cocaína com cerca de 870 mil usuários da América do Sul. Pesquisas domiciliares realizadas pelo governo constataram que a prevalência anual do uso de pelo menos uma vez no ano passou de 0,4\% da população entre 12 e 65 anos em 2001, para 0,7\% em 2005. As regiões Sudeste e Sul do Brasil são as áreas mais afetadas pelo consumo de cocaína, e, consequentemente, a disponibilidade e o tráfico desta droga é maior nessas regiões. ${ }^{8}$

Tabela 4 - Tipos de drogas de primeiro uso pelos dependentes químicos da unidade de reabilitação de adictos. Paraná, 2008

\begin{tabular}{|c|c|c|c|c|c|c|c|c|}
\hline \multirow{2}{*}{ Droga } & \multicolumn{2}{|c|}{ Álcool } & \multicolumn{2}{|c|}{ Maconha } & \multicolumn{2}{|c|}{ Tabaco } & \multicolumn{2}{|c|}{ Total } \\
\hline & \multirow[t]{2}{*}{$\%$} & \multirow{2}{*}{ (n) } & \multirow[t]{2}{*}{$\%$} & \multirow{2}{*}{ (n) } & \multirow[t]{2}{*}{$\%$} & \multirow{2}{*}{ (n) } & \multirow[t]{2}{*}{$\%$} & \multirow{2}{*}{ (n) } \\
\hline Idade & & & & & & & & \\
\hline Abaixo dos 12 anos & 10 & (3) & & & 6 & (2) & 16 & (5) \\
\hline Entre 13 e 19 anos & 48 & (14) & 3 & (1) & 10 & (3) & 61 & (18) \\
\hline Entre 20 e 35 anos & 10 & (3) & & & & & 10 & (3) \\
\hline Entre 36 e 55 anos & 3 & (1) & 10 & $(3)$ & & & 13 & (4) \\
\hline Total & 71 & (21) & 13 & (4) & 16 & (5) & 100 & (30) \\
\hline
\end{tabular}

Fonte: Da pesquisa.

Como mostra a Tabela 4, que $71 \%(n=21)$ dos participantes iniciaram o uso de drogas pelo álcool e a maioria, $58 \%(n=17)$, na faixa etária de 12 a 19 anos; 0 uso dessa droga foi de maior prevalência em todas as faixas etárias. Nessa idade 0 efeito do álcool ou qualquer outro tipo de droga é extremamente maléfico, pois altera a memória, ritmo do sono, causa déficit de atenção e irritabilidade naqueles que 0 usam uma vez que compromete a vida escolar e afetiva dos usuários dessafaixa etária. ${ }^{2}$ Com idade inferior a 12 anos, $10 \%(n=3)$ dos sujeitos afirmaram que iniciaram o uso de drogas e utilizaram o álcool como primeira droga e $6 \%(n=2)$ referiram que o início foi pelo tabaco. Na faixa etária entre 13 e 19 anos, $48 \%$ ( $n=14)$ referiram ter utilizado o álcool como primeira droga. Entre 20 e 35 anos, 10\% $(n=3)$ iniciaram com álcool, e entre 35 e 55 anos, $3 \%(n=1)$ iniciaram utilizando o álcool como primeira droga.
A respeito de sua iniciação com a droga, $71 \%(n=21)$ dos dependentes químicos afirmaram que foi no meio familiar com o uso de álcool e tabaco e, em 30\% ( $n=9$ ) dos casos, foi por meio de amigos. Verificou-se também que $70 \%(n=21)$ dos dependentes possuem na família um outro dependente químico.

Observa-se um paradoxo acerca desta questão ao considerarmos que a família assume papel importante no tratamento da dependência química e, em conjunto com o dependente químico, deve participar do processo de tratamento, prevenção da recaída, enfim, da luta contra o vício. Entretanto, é na família que ocorre o maior índice de iniciação ao uso da droga.

Ressalva-se que na prática assistencial ao dependente químico, indivíduos que mantêm vínculo familiar no momento e 
durante a internação têm um maior aproveitamento do tratamento do que aqueles que não possuem família; isso é constatado na manutenção do tratamento da dependência química. ${ }^{16}$

Nesta pesquisa, $71 \%(n=21)$ dos participantes afirmaram que em sua infância existia um dependente químico na família ou muito próximo dele. 0 número de drogaditos que possuem algum familiar dependente químico é superior àqueles que não têm histórico familiar de uso de drogas. Considera-se fator de risco para dependência química uma criança ou jovem que convive com alguém que utiliza de maneira abusiva algum tipo de substância psicoativa. ${ }^{12}$ É importante lembrar que o filho, ao ver o pai utilizar áàlcool ou tabaco, torna-se um sujeito com elevadas possibilidades de repetir esse ato durante sua vida. ${ }^{16}$

Filhos de dependentes químicos ou aqueles que convivem próximo ao dependente apresentam risco aumentado para outros transtornos psiquiátricos, desenvolvimento de problemas físicoemocionais e dificuldades escolares. Esses apresentam um risco aumentado para o consumo de substâncias psicoativas, quando comparados com filhos de não dependentes químicos, sendo que filhos de alcoolistas têm um risco aumentado em quatro vezes para o desenvolvimento do alcoolismo. ${ }^{12}$

\section{CONCLUSÃO}

Pelos dados apresentados e discutidos, pode-se considerar que as drogas afetam de várias formas a vida das pessoas, uma vez que as perdas pessoais e sociais fazem parte do cotidiano do indivíduo dependente de substâncias psicoativas e, por consequência, repercutem na vida familiar.

Destarte, os profissionais da saúde precisam ser qualificados e buscar desenvolver ações de prevenção, as quais devem se constituir o foco das políticas de saúde relacionadas a essa temática, pois o consumo de drogas é um problema de saúde pública e afeta a sociedade de maneira geral. Porquanto, é imprescindível que as ações de prevenção ao uso de substâncias psicoativas sejam articuladas entre atenção básica, instituições de educação básica e envolvam a família e a sociedade.

Sugere-se que sejam realizados mais estudos desta natureza, a fim de aprofundar a temática e subsidiar as ações de promoção da saúde, prevenção e tratamento dos dependentes

\section{REFERÊNCIAS}

1. Roehrs H, Lenardt MH, Maftum MA. Práticas culturais familiares e o uso de drogas psicoativas pelos adolescentes: reflexão teórica. Esc Anna Nery Rev Enferm. 2008 jun; 12(2): 353-57.

2. Louzã MRN, Hélio E. Psiquiatria básica. $2^{\mathrm{a}}$ ed. Porto Alegre (RS): Artmed; 2007.
3. Funchal E, Lenardt MH, Labronici LM, Polak YSN. Rituais vivenciados pelo corpo fumante. Cogitare Enferm 2004 jan/abr; 10(1): 53-9.

4. Stuart GW, Laraia MT. Enfermagem psiquiátrica: princípios e prática. Tradução de Dayse Batista. $6^{a}$ ed. Porto Alegre(RS): Artmed; 2001.

5. Miranda FAN, Azevedo DM, Santos RA, et al. Predisposição ao uso e abuso de álcool entre estudantes de graduação em Enfermagem da UFRN. Esc Anna Nery Rev Enferm 2007 dez; 11 (4): 663-69.

6. Ministério da Saúde (BR). Secretaria de Atenção à Saúde. A política do Ministério da Saúde para a atenção integral a usuários de álcool e outras drogas. Brasília(DF); 2004.

7. Ferreira FGKY, Luz AJ, Obrzut-Neto L, Santos KA. Uma visão multiprofissional humanizada no tratamento da pessoa com dependência química em enfermaria psiquiátrica de um hospital geral no Paraná. Cogitare Enferm 2005 maio/ago; 10(2): 54-62.

8. Organização das Nações Unidas-OMS. Programa para o Controle Internacional de Drogas. Escritório das Nações Unidas Contra Drogas e Crime-UNODOC. Brasília(DF); 2008. [citado 15 out 2008]. Disponível em: http://www.unodc.org/brazil/pt/pressrelease_20080626.html

9. Araújo RB, Souto KEP. Repercussões do fechamento da unidade de desintoxicação do hospital psiquiátrico São Pedro. Rev Psiquatr RS 2002 maio/ago; 24(2): 149-54.

10. Ministério da Saúde (BR). Conselho Nacional de Saúde. Resolução n. ${ }^{\circ}$ 196, de 10 de outubro de 1996. Diretrizes e normas regulamentadoras de pesquisa envolvendo seres humanos. Brasilia (DF); 1996.

11. Resende G, Amara V, Bandeira M, Gomide A, Andrade E. Análise da prontidão para o tratamento em alcoolistas em um centro de tratamento. Rev Psiquiatr Clin 2005 jul./ago; 32(4): 211-17.

12. Figlie N, Fontes A, Moraes E, Payá R. Filhos de dependentes químicos com fatores de risco bio-psicossociais: necessitam de um olhar especial? Rev Psiquiatr Clin 2004; 31(2): 52-62

13. Heim J, Andrade AG. Efeitos do uso do álcool e das drogas ilícitas no comportamento de adolescentes de risco: uma revisão das publicações científicas entre 1997 e 2007. Rev Psiquiatr Clín 2008; 35(supl1): 61-64.

14. Werneck G, Hasselmann M, Phebo L, Vieira D, Gomes V. Tentativas de suicídio em um hospital geral no Rio de Janeiro, Brasil. Cad Saúde Pública 2006 out; 22(10): 2201-206.

15. Brasil. Ministério da Saúde. Secretaria de Atenção à Saúde. Área de Saúde do Adolescente e do Jovem. - Marco legal: saúde, um direito de adolescentes. Ministério da Saúde. (Série A. Normas e Manuais Técnicos), Brasília; 2005.

16. Knapp P. Terapia cognitivo-comportamental na prática psiquiátrica. Porto Alegre(RS): Artmed; 2004. 